\title{
Purtscher-like retinopathy in a 56-year-old Thai female with anti-glomerular basement membrane glomerulonephritis
}

\author{
Supalert Prakhunhungsit, Rossukon Khotcharrat \\ Department of Ophthalmology, Faculty of Medicine, Naresuan University, Thailand
}

\begin{abstract}
Purpose: To report a case of Purtscher-like retinopathy in an anti-glomerular basement membrane glomerulonephritis in a 56-year-old Thai female.

Method: Observational case report.

Results: A 56-year-old Thai female was referred to Naresuan university hospital with anemia and pitting edema in both legs. She had underlying diseases of hypertension, hypercholesterolemia and chronic renal failure with anemia. Before her referral, she was treated by an internist at a provincial hospital. Because of deterioration of creatinine level and anemic symptoms, she was advised to see a nephrologist for the diagnosis of causative pathology and treatment. A re-evaluation of chronic renal failure was done. The results of laboratory investigation were creatinine level of $2.42 \mathrm{mg} / \mathrm{dl}$, blood urea nitrogen (BUN) $44 \mathrm{mg} / \mathrm{dl}$ and the urinalysis found microscopic hematuria. Further evaluations for hematuria; vaginoscopy, cystoscopy and ultrasonography of genitourinary system were performed, but the outcome was normal. The result of a renal biopsy was anti-glomerular basement membrane glomerulonephritis. Hemodialysis and total plasma exchange were done several times to reduce the circulating antibody of the anti-glomerular basement membrane level.

During her admission, the patient complained of sudden blurred vision in both eyes after the ninth total plasma exchange. She had no previous ocular problems nor physical trauma. The ocular examination showed visual acuity of counting fingers in both eyes. Anterior segment was normal for her age. The posterior segments showed retinal thickening around the posterior poles with clear intervening zones in both eyes with some intra-retinal hemorrhage, which was consistent with the earlier-diagnosed Purtscherlike retinopathy. The pathogenesis of Purtscher-like retinopathy was investigated with negative results. During counseling, the patient and her family chose observation instead of active treatment. The patient was discharged and followed-up at the out-patient unit of the ophthalmology department of the Naresuan university hospital.

Conclusion: Purtscher-like retinopathy is a rare ocular disease with unknown pathogenesis. There are many diseases reported to be associated with Purtscher-like retinopathy. In this case, we found Purtscher-like retinopathy in a patient with anti-glomerular basement membrane glomerulonephritis.
\end{abstract}

Key words: Purtscher-like retinopathy; Anti-Glomerular Basement Membrane Glomerulonephritis; Total plasma exchange

Correspondence: Supalert Prakhunhungsit, MD, Department of Ophthalmology, Faculty of Medicine, Naresuan university hospital, Naresuan university, Thailand 65000. E-mail: off sp25@hotmail.com 


\section{Introduction}

Purtscher's retinopathy is a rare ophthalmologic disease. It was first described by Otmar Purtscher in 1910, in a patient with a history of head trauma resulting from falling from a tree. ${ }^{1}$ The patient showed retinal whitening and retinal hemorrhage around the posterior pole of both eyes. Causes other than trauma, with the same ophthalmic findings are diagnosed as Purtscher-like retinopathy. The most common cause of Purtscher-like retinopathy is acute pancratitis. ${ }^{2,3}$ Long-bone fracture, connective tissue diseases such as systemic lupus erythromatosus (SLE), chronic renal failure and orbital anesthetic injection are also reported to be causes of Purtscher-like retinopathy. ${ }^{2,3}$ The main pathogenesis is thought to be associated with micro-embolization of the retinal arterioles from various causes. ${ }^{2-7}$ The pathogenesis of hyperviscosity and vascular autoregulation are now under investigation. ${ }^{8,9}$ Recently, computational fluid dynamic simulation software has shown the role of wall shear stress as a new mechanism in the development of Purtscher's and Purtscher-like retinopathies. ${ }^{10}$ Treatment of Purtscher-like retinopathy is to correct the causative systemic pathology and for ocular treatment, observation is usually advised. ${ }^{2}$ The visual prognosis of Purtscher-like retinopathy is guarded.

Anti-glomerular basement membrane glomerulonephritis is a rare kidney disease with an incidence of one case per million. ${ }^{11}$ The affected patients usually come in with symptoms of acute or sub-acute renal failure with varied clinical forms from mild severity to rapidly progressive glomerulonephritis (RPGN). The anti-glomerular basement membrane antibody circulates in the serum of the patients and destroys the basement membrane of the renal glomeruli. ${ }^{11,12}$ The antibody affects collagen type IV, which is the composition of the renal glomerular basement membrane. In some cases, the antibody also affects the respiratory system. Because of the same collagen component, it causes alveolar hemorrhage. ${ }^{13}$ Renal failure combined with alveolar or pulmonary hemorrhage is called Goodpasture's syndrome. Due to the circulating antibody in the patient's blood stream, the treatment modalities of antiglomerular basement membrane glomerulonephritis are plasmapheresis, corticosteroids, and immunosuppressive drugs. Plasmapheresis is used for the reduction of the circulating anti-glomerular basement membrane antibody found in the patient's plasma. ${ }^{11}$

\section{Material and methods}

The patient was referred from a provincial hospital to the Naresuan university hospital with an increasing level of serum creatinine and deterioration of anemic symptom. The history and physical findings were reported. The patient was re-evaluated because of the sub-acute renal failure. Special investigations, such as renal biopsy, were done and reported. The renal pathological specimens were shown and detailed in this report. The ocular diagnosis and differential diagnosis of its cause were detailed through the treatment of the patient. 


\section{Case history}

A 56-year-old Thai female with underlying diseases of essential hypertension, hypercholesterolemia and the recent diagnosis of chronic renal failure for a few months prior to her referral. She was followed-up and treated at a provincial hospital by an internist. Medication consisted of amlodipine, enalapril, furosemide, hydralazine, gemfibrozil, aspirin, folic acid and calcium supplement. In this followup, an increasing level of serum creatinine was found, up to the level of $2.42 \mathrm{mg} / \mathrm{dl}$ and her hematocrit dropped from $26 \%$ to $20 \%$ without blood loss. She also experienced mild dyspnea from chronic anemia and increasing swelling of her legs from chronic renal failure. She was referred to a nephrologist for further evaluation and treatment at Naresuan university hospital.

The vital signs were within normal limits; blood pressure $107 / 65 \mathrm{mmHg}$, pulse rate $75 / \mathrm{min}$. The physical examination showed a moderately pale conjunctiva and moderately pitting edema in both legs. The medication was adjusted and the followup was done at Naresuan university hospital by a nephrologist. Re-investigation showed hematocrit $24.2 \%$, blood urea nitrogen (BUN) $20.3 \mathrm{mg} / \mathrm{dl}$, creatinine 2.4 $\mathrm{mg} / \mathrm{dl}$. Urinalysis results showed proteinuria 1+, and red blood cells (RBC) 50-100 cells/HPF. The hematocrit, blood urea nitrogen and creatinine levels were compatible with the diagnosis of chronic renal failure/chronic kidney disease stage IV, but the microscopic hematuria was not explained. The urinalysis was redone with the same findings, so she was sent to evaluate the cause of microscopic hematuria due to its possibility to correlate with the cause of her worsening signs of chronic kidney disease. A vaginoscopy was done with the result of normal mucosa and no bleeding. The cystoscopy result was normal without blood or bladder stone(s). The ultrasonography of genitourinary system was normal. A renal biopsy was done after follow-up without the improvement of microscopic hematuria. The result of the renal biopsy with the immunofluorescent study showed positive linear staining for lgG (3+) along glomerular basement membrane with foci of breaks in glomerular basement membrane, presence of fibrocellular and fibrous crescent. The circulating antibody was also found in the patient's blood circulation, with titer of 1:160. All these findings were compatible with the diagnosis of anti-glomerular basement membrane glomerulonephritis. 


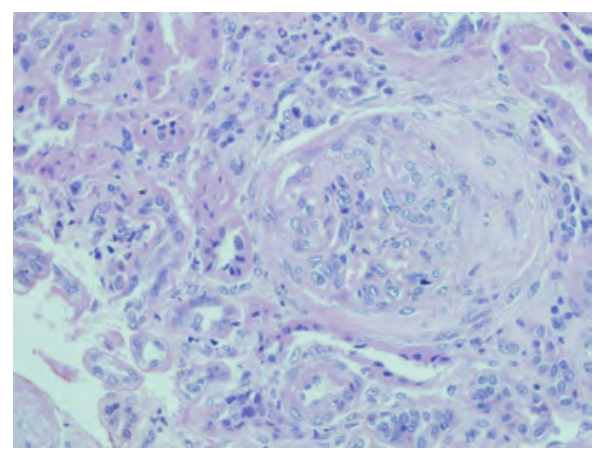

Fig. 1. H-E 40x: Hematoxylin and eosin stain, 40x, fibrocellular crescent of glomerulus.

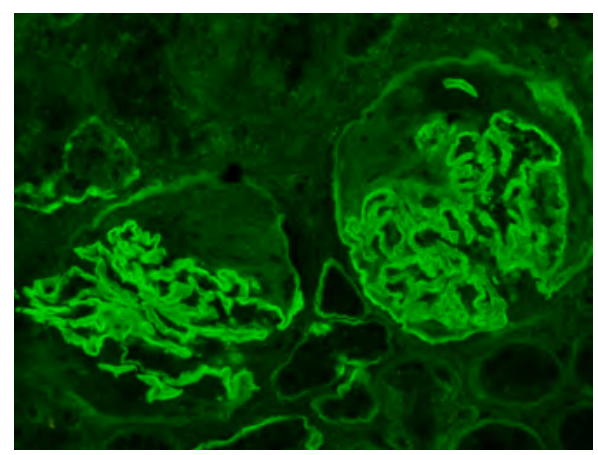

Fig. 3. Ig G-40x-2: Immunofluorescent IgG antibody, 40x, positive 3+ along GBM.

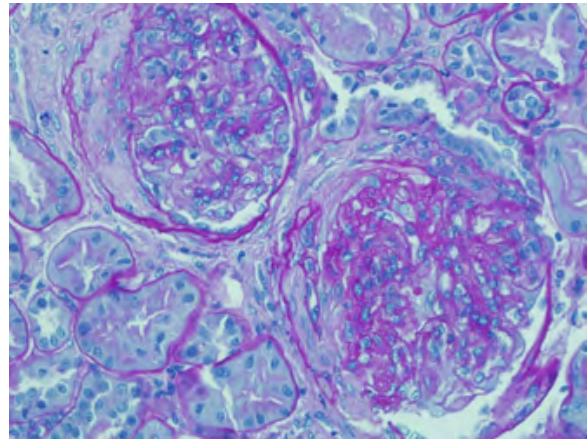

Fig. 2. PAS-40x : Periodic acid schiff stain 40x, fibrocellular crescent of glomeruli.

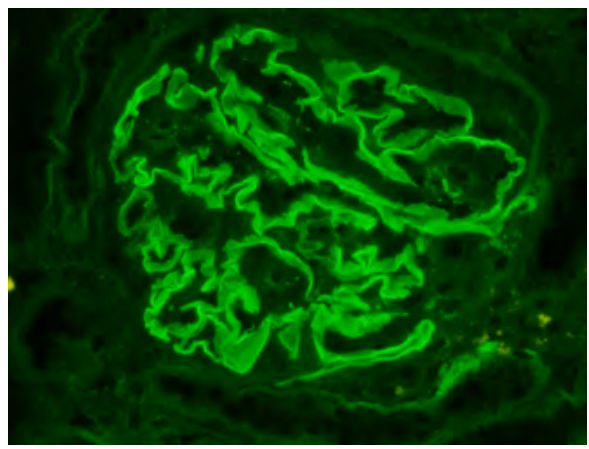

Fig. 4. IgG-60x: Immunofluorescent IgG antibody, 60x, positive 3+ along GBM.

The treatment for the patient was pulse corticosteroids (methylprednisolone 750 $\mathrm{mg}$ intravenously for three days) and cyclophosphamide (100 mg/day). There was no improvement of her condition and circulating antibody level after the treatment was started, so plasmapheresis was advised. The patient underwent total plasma exchange (TPE) ten times and after the sixth episode of TPE the antibody titer had reduced from 1:160 to 0 .

The patient started complaining of blurred vision in both eyes after the ninth TPE, and was referred to the ophthalmology department. Ocular examination found visual acuity of counting fingers in both eyes, no relative afferent pupillary defect, nuclear sclerotic cataract in both eyes, no anterior chamber cells and flare. The fundoscopic findings were whitening of retina around posterior pole with clear intervening zones between the retinal arterioles compatible with Purtscher flacken, which is pathognomonic for Purtscher-like retinopathy, some retinal hemorrhages, and cotton wool spots in both eyes. No perivascular sheathing. No vitreous cells. Optic disc margins were not swollen and not pale. Based on these findings, she was diagnosed as Purtscher-like retinopathy. Optical coherent tomography (OCT) 
of both eyes was done and retinal thickening around posterior pole and macular edema were found. Because she had already received a high dose of corticosteroids which was one of the reported treatment choices for the Purtscher-like retinopathy, the patient did not want any more treatments for her ocular problems. Special investigations, such as fundus fluorescent angiography (FFA), were discussed during treatment counseling, but the patient refused because of severe dizziness and her impaired renal function. The follow-up of her ocular symptoms was performed at the out-patient department of the ophthalmology department of the Naresuan university hospital.

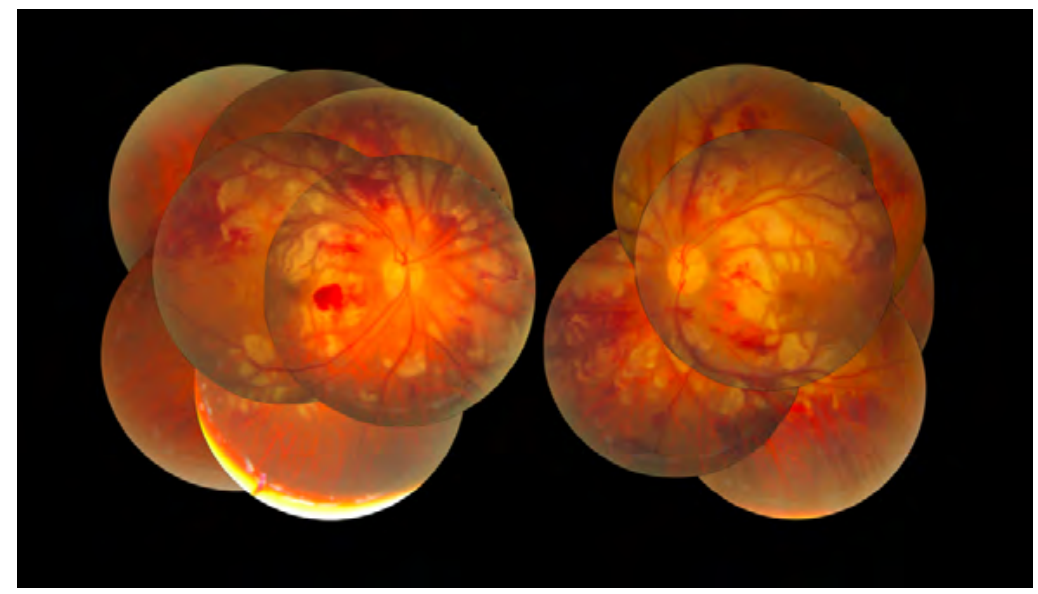

Fig. 5. Color fundus photography of both eyes showed whitening of the retina around the posterior pole with clear intervening zones between the retinal arterioles, retinal hemorrhages, and cotton wool spots. Disc margins were not swollen.

The first follow-up visit took place two months after the first diagnosis. There was improvement of the retinal thickening at the posterior poles in both eyes. Vision in both eyes was not significantly improved, the final visual acuity (BCVA) was $6 / 200,3 / 200$. Optic discs were pale with the attenuation of retinal vessels.

The laboratory findings of circulating autoantibody was negative after the sixth of the ten total plasma exchanges that were performed during her admission. The hemodialysis was continued with the protocol of a chronic kidney disease patient. The immunosuppressive agents were continued; oral prednisolone and cyclophosphamide were administered. 


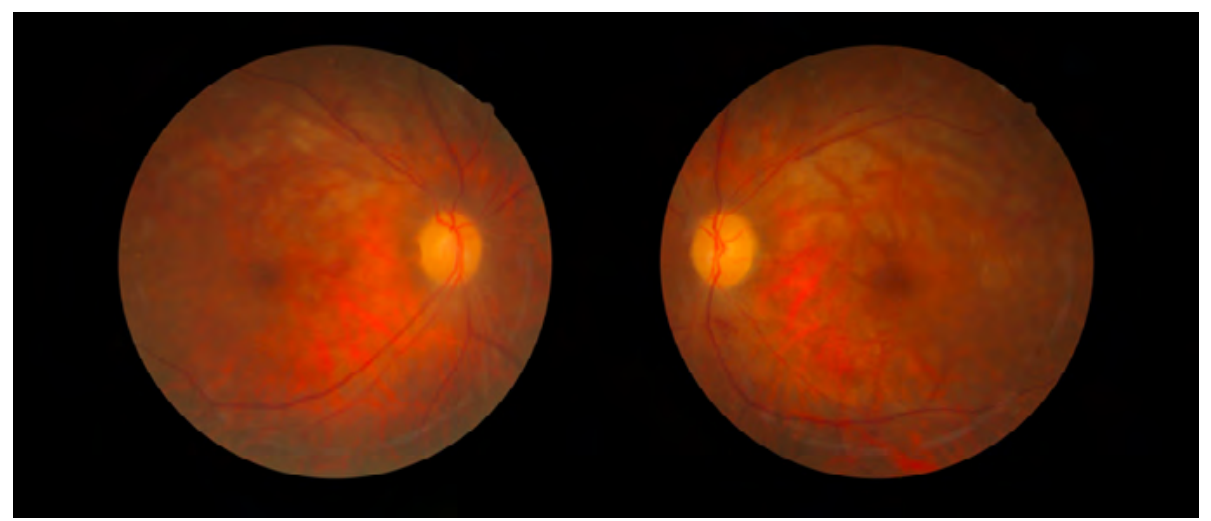

Fig. 6. Color fundus photography of both eyes (two months after the first diagnosis) showed improvement of the thickened retina around the posterior poles, less retinal hemorrhages, and cotton wool spots. Optic discs were mildly pale without swelling of the margins.

\section{Discussion}

This patient had already been diagnosed of renal insufficiency and microscopic hematuria. She was evaluated and treated by a nephrologist, but her condition did not improve despite the proper treatment. Also, the cause of microscopic hematuria was not explained by her underlying diseases. Therefore, a renal biopsy was done to investigate the cause of microscopic hematuria. The renal biopsy result revealed the underlying pathology of anti-glomerular basement membrane glomerulonephritis. The proper treatment was advised and the choice of plasmapheresis was given together with intravenous and oral corticosteroids and immunosuppressive drugs. After the ninth episode of total plasma exchange (TPE), the patient complained of acute blurred vision. The further review of the last episode of TPE was normal, without any complications. Ocular examination showed a visual acuity of counting fingers at three feet in both eyes. The anterior segment showed senile nuclear sclerotic cataracts which did not explain the cause of her visual loss. The relative afferent pupillary defect was negative. The fundoscopic findings were retinal whitening around the posterior pole with clear intervening zones in both eyes which was compatible with Purtscher fleckens, cotton wool spots and retinal hemorrhage in both eyes. All of the described fundoscopic findings were consistent with the diagnosis of Purtscher's retinopathy. Without the obvious history of head or chest trauma, the diagnosis of Purtscher-like retinopathy was made.

The most common cause of Purtscher-like retinopathy is acute pancreatitis, ${ }^{2}$ of which there was no clinical sign in this patient. From the systemic review of history and physical examination of this patient, there was only the diagnosis of antiglomerular basement membrane glomerulonephritis with anemia, essential hypertension and hypercholesterolemia. As far as the authors know, there was no report of an association of anti-glomerular basement membrane glomerulonephritis and Purtscher-like retinopathy. ${ }^{2,3}$ 
Anti-glomerular basement membrane glomerulonephritis is an auto-immune disease caused by $\lg G$ autoantibodies against the glomerular basement membrane. It shows the clinical picture of renal insufficiency from mild severity to rapidly progressive glomerulonephritis (RPGN). With the same collagen type IV of glomeruli, it also affects the alveoli of the lungs and causes pulmonary hemorrhage (Goodpasture's syndrome). The presence of circulating auto-antibody level is needed for the immunological diagnosis of anti-GBM glomerulonephritis and its level is directly related to the disease severity. ${ }^{11}$ The combination of renal and pulmonary systems is not always presented, there is a group of patients that referred to the clinic only for renal problems. In our patient, for determination of the cause of the microscopic hematuria, normal vaginoscopy and normal cystoscopy were performed. The renal ultrasonography was normal with normal size of both kidneys. After the renal biopsy (Figs. 1 and 2), the pathological findings showed the positive linear staining for $\operatorname{lgG}(3+)$ along the glomerular basement membrane with foci of breaks in the glomerular basement membrane (Figs. 3 and 4). The result suggested anti-glomerular basement membrane glomerulonephritis. The circulating autoantibody to glomerular basement membrane was positive with titer of 1:160. Consequently, the definite diagnosis of anti-glomerular basement membrane glomerulonephritis was made.

In experiments, the circulating microparticle of glass sphere, fat, platelet and leukocyte aggregates were injected in pigs' and cats' circulation, the intermediate sized of microparticles can cause the clinical picture of Purtscher-like retinopathy. ${ }^{2-7}$ In anti-glomerular basement membrane glomerulonephritis the circulating particles or antigen-antibody complex are also found, ${ }^{11,12}$ which can explain the pathophysiology of Purtscher-like retinopathy in our patient. However, as far as the author's knowledge, there is no previous report showing the association of these two diseases. This paper is the first report showing some association between Purtscher-like retinopathy and anti-glomerular basement membrane glomerulonephritis. The definite conclusion of the pathophysiology of the patient with antiglomerular basement membrane glomerulonephritis with Purtscher-like retinopathy has to be further studied.

Our patient developed blurred vision after the ninth total plasma exchange before the Purtscher-like retinopathy was diagnosed. W.H. Reinhart et al. reported the change of hemorheology of the circulation after hemodialysis with the result of increased hematocrit and increased plasma and whole blood viscosity after the hemodialysis was done, due to the influence of the hemodialysis passage. ${ }^{9}$

Amit Nautiyal et al. reported a case of patient with Purtscher-like retinopathy with multiple myeloma. ${ }^{8}$ In their case, hyperviscosity from multiple myeloma was mentioned to be the cause of Purtscher-like retinopathy. Then, the several times of total plasma exchanges in our patient could lead to the plasma hyperviscosity and thus, Purtscher-like retinopathy. 


\section{Conclusion}

Purtscher-like retinopathy is a rare ocular disease with unknown pathogenesis. There are some systemic diseases which are known to be associated with Purtscherlike retinopathy, but there is no report about anti-glomerular basement membrane glomerulonephritis. This is the first case report of Purtscher-like retinopathy in anti glomerular basement membrane glomerulonephritis patient. The pathogenesis could be from the circulating autoantibody by the proposed mechanism of micro-embolisation. However, the hemodynamic and fluidic change after plasma exchange might also play a role in development of Purtscher-like retinopathy in this case. The mechanism of Purtscher-like retinopathy occurred in chronic renal failure could also be in differential of pathogenesis. Further study is needed to confirm the mechanism and possibility of the association. In complicated cases without obvious causes, the pathogenesis of the disease should be investigated which directs to the proper treatment and better visual prognosis.

\section{Acknowledgement}

La-or Chompuk, MD

Department of Pathology, Faculty of Medicine, Naresuan University, Thailand

\section{References}

1. Otmar P. Noch unbekannte Befunde nach Schaedel-trauma. Ber Dtsch Ophthalmol Ges 1910;36:294-301.

2. Miguel AIM, Enriques F, Azevedo JFR, Loureiro AJR, Maberley DAL. Systematic review of Purtscher's and Purtscher-like retinopathies. Eye 2013;27:1-13.

3. Agrawal A, McKibbin MA. Purtscher's and Purtscher-like retinopathies: A Review. Surv Ophthalmol 2006;51(2):129-136.

4. Holak H, Holak N, Huzarska M, Holak S. Pathogenesis of Purtscher's retinopathy and Purtscher-like retinopathy. Klin Oczna 2007;109(1-3):38-45.

5. Lai J, Johnson MW, Martonyi C, Till GO. Complement-induced retinal arteriolar occlusions in the cat. Retina 1997;17(3):239-246.

6. Dollery CT, Henkind P, Paterson JW, Ramalho PS, Hill DW. Ophthalmoscopic and circulatory changes in focal retinal ischemia. Brit J Ophthalmol 1966;50:285.

7. Scheurer G, Praetorius G, Damerau B, Behrens-Baumann W. Vascular occlusion of the retina: an experimental model. Graefe's Archive Ophthalmology 1992;230(3):275-280.

8. Nautiyal A, Amescua G, Jameson A, Gradowski JF, Hong F, Doft B. Sudden loss of vision: Purtscher retinopathy in multiple myeloma. Can Med Ass J 2009;181(12).

9. Reinhart WH, Cagienard F, Schulzki T, Venzin RM. The passage of a hemodialysis filter affects hemorheology, red cell shape, and platelet aggregation. Clin Hemorheol Microcirc 2014;57:49-62.

10. Harrison TJ, Abbast CO, Khraishi TA Purtscher Retinopathy: An Alternative Etiology Supported by Computer Fluid Dynamic Simulations. Invest Ophthalmol Vis Sci 2011;52(11):8102-8107.

11. Lahmer T, Heemann U. Anti-glomerular basement membrane antibody disease: A rare autoimmune disorder affecting the kidney and the lung. Autoimmun Rev 2012;12:169-173.

12. Pusey CD. Anti-glomerular basement membrane disease. Kidney International 2003;64:1535-1550.

13. Dammacco F. Goodpasture's disease: A report of ten cases and a review of the literature.

Autoimmun Rev 2013;12:1101-1108. 